\title{
Superconducting Properties and Critical Current Density of Nb-Ti/Ti Multilayers
}

\author{
L. D. Cooley, C. D. Hawes, P. J. Lee, and D. C. Larbalestier \\ Applied Superconductivity Center, University of Wisconsin, 1500 Engineering Drive, Madison, WI 53706
}

\begin{abstract}
Multilayers of $\mathrm{Nb47Ti}$ superconductor (S) and titanium pins (N) have been made with a critical temperature $T_{c}$ and an upper critical field $\boldsymbol{H}_{c 2}$ approaching bulk values. There is no proximity effect suppression of $T_{c}$ and $H_{c 2}$ for layer thickness $d_{S}=d_{N}=10 \mathrm{~nm}$, in contrast to the strong suppression for comparable multilayers made with $\mathrm{Cu}, \mathrm{Cu}$-alloy, or $\mathrm{Nb}$ pins. This may be because the proximity length of the $T i$ layers, $\sim 10$ $\mathrm{nm}$, is less than or equal to their thickness. The critical current density $J_{c}$ exhibits multiple peaks in $J_{c}(H)$, which suggests that matching effects contribute to the overall pinning. $J_{c}$ for $20 \mathrm{~nm}$ bilayers is comparable to that of Nb47Ti tapes, which have a higher number density of pins but a lower pin volume fraction. The weak proximity coupling suggests that much higher $J_{c}$ can be obtained if smaller bilayer periods can be made with good adhesion. The results are compared to other $\mathrm{Nb}-\mathrm{Ti} / \mathrm{Ti}$ multilayer experiments, and stability limitations are also discussed.
\end{abstract}

\section{INTRODUCTION}

Niobium-titanium alloy composites attain strong flux pinning and high critical current density $J_{c}$ when a nanostructure of the superconducting and non-superconducting phases is made. In conventional wires, non-superconducting $\alpha$-Ti precipitates are the pins and have $1-4 \mathrm{~nm}$ thickness and 4-10 nm separation when flux pinning is optimum [1]. In artificial pinning-center (APC) composites with $\mathrm{Nb}$ pins, the optimum nanostructure is somewhat less refined, the pins having 10-15 $\mathrm{nm}$ thickness and 10-30 $\mathrm{nm}$ separation [2]. Both conventional and APC composites also exhibit proximity effects when the optimum nanostructure is obtained, characterized by changes in the critical temperature $T_{c}$ and the upper critical field $H_{c 2}$ [2]-[4]. The differences in the optimum flux-pinning states and the connection to the proximity effect were explained by Cooley et al. [2], [5] in terms of a magnetic pinning mechanism, where the proximity length $\xi_{N}$ is thought to be the parameter that determines the optimum nanostructural state.

In both conventional and APC composites, the pins and the superconductor take the form of ribbon-like regions that are folded and curled together [1]-[3]. Thus, multilayers of Nb$T i$ and various pin materials have been used to model the pinning force and separate out the effects of orientation of the pin to the field [6]-[8]. Multilayers also give the flexibility to vary the pin material without concern for ductility and other fabrication issues. Multilayers of $\mathrm{Nb} 47 \mathrm{Ti}$ with $\mathrm{Cu}$ pin layers exhibited $J_{c} \sim 4 \mathrm{kA} / \mathrm{mm}^{2}$ at $5 \mathrm{~T}$ parallel field and $4.2 \mathrm{~K}$ [6],

Manuscript received September 15, 1998.

This work was supported by the U.S. Department of Energy, Division of High-Energy Physics, under contract \#DE-FG02-96ER40961, and by the NSF Materials Research Science and Engineering Center for Nanostructured Materials and Interfaces.
[9], and they had an upper critical field of $11 \mathrm{~T}$, even though $T_{c}$ was suppressed. However, efforts to further increase $J_{c}$ by alloying the pin layer and reducing $\xi_{N}$ resulted in a greater suppression of superconducting properties [10]. Multilayers with $\mathrm{Nb}$ pin layers have been extensively explored because they exhibit no suppression of $T_{c}$ and interesting properties of $H_{c 2}$ [7], [11]. In this case $J_{c}$ is $\sim 10 \mathrm{kA} / \mathrm{mm}^{2}$ [7], although the field dependence $J_{c}(H)$ is not monotonic and $H_{c 2}$ is suppressed. Multilayers with $\mathrm{Ti}$ pins can achieve $J_{c}>20$ $\mathrm{kA} / \mathrm{mm}^{2}$ at $5 \mathrm{~T}$ parallel field and $4.2 \mathrm{~K}[7]$. This is a factor of 4 higher than the $J_{c}$ of a $\mathrm{Nb}-\mathrm{Ti}$ tape with $\alpha-\mathrm{Ti}$ precipitates aligned to within $\sim 10^{\circ}$ of the tape face [12]. This result is intriguing because the bilayer period $\Lambda=d_{S}+d_{N}$ of the multilayer in [7] is somewhat larger than the average precipitate spacing in optimized flattened conventional composites [12], $\sim 20$ vs. $\sim 5-10 \mathrm{~nm}$. Further increase of $J_{c}$ may thus come if the pin thickness $d_{N}$ and the superconductor thickness $d_{S}$ can be reduced to a few nanometers. However, the presence of thick $\mathrm{Nb}$ buffer layers masked the superconducting properties of that multilayer, and there is little other characterization to explain this singular result.

The superconducting properties and critical current density of multilayers of $\mathrm{Nb} 47 \mathrm{Ti}$ superconductor $(\mathrm{S})$ and $\mathrm{Ti}$ pin $(\mathrm{N})$ layers are examined in this paper. Superconducting properties close to those of bulk specimens were obtained for thin films of $\mathrm{Nb} 47 \mathrm{Ti}$, as is discussed more completely in another paper [13]. These properties are maintained in the multilayers by using the same deposition parameters as for the films. A surprising result is $T_{c}=8.6 \mathrm{~K}$ and $\mu_{0} H_{c 2}=11.4$ $\mathrm{T}$, even for $d_{S}=d_{N}=10 \mathrm{~nm}(10 / 10)$, about twice the coherence length $\xi=\left(\phi_{0} / 2 \pi \mu_{0} H_{c 2}\right)^{1 / 2} \approx 5 \mathrm{~nm}$. This suggests that the proximity effect is weak, perhaps because $\xi_{N}$ is $\sim 10$ $\mathrm{nm}$, as deduced from the resistivity. The superconducting layers then appear to behave independently when $d_{N}=15 \mathrm{~nm}$ $>\xi_{N}$, where peaks in $J_{c}(H)$ suggest the penetration of fluxline rows into the $S$ layer when $d_{S}$ or $\Lambda$ is a multiple of the flux-line spacing $a_{0} \approx\left(\phi_{0} / \mu_{0} H\right)^{1 / 2}$. Similar phenomena are found for the $10 / 10$ multilayer, where $J_{c}$ has 2 plateaus and drops from $\sim 8 \mathrm{kA} / \mathrm{mm}^{2}$ at $4 \mathrm{~T}$ to $\sim 5 \mathrm{kA} / \mathrm{mm}^{2}$ at $5 \mathrm{~T}$ and $\sim 2$ $\mathrm{kA} / \mathrm{mm}^{2}$ at $6 \mathrm{~T}$ parallel field, when $a_{0} \approx \Lambda$. The $J_{c}$ values for the 10/10 multilayer are comparable to the tape results in [12], but for about one-half the pin number density.

Because of the weak proximity effect, further increase in $J_{c}$ should thus be possible if $1-5 \mathrm{~nm}$ layers can be made and the associated adhesion problems solved [13]. Transmission electron microscopy (TEM) shows that the layers are perturbed by columnar grain growth through several layers at this length scale. Although the matching effects and other data suggest that the lack of layer uniformity does not adversely affect flux pinning, the grains may trap gases present during sputtering, which adds stress to the multilayer. Poor adhesion has so far prevented exploration of smaller bilayer periods. Other limitations, such as poor stability of the multilayer, are also discussed. The present work is also 
compared to [7] and several important differences between the experiments are discussed.

\section{EXPERIMENT}

The multilayers were fabricated by dc sputtering at room temperature on R-plane sapphire, using a technique described previously [6]. A high-homogeneity alloy target was used for the superconductor. The deposition rates were 2.8 and 2.0 $\mathrm{nm} / \mathrm{s}$ for S and $\mathrm{N}$ respectively, at a power density of about 13 $\mathrm{W} / \mathrm{cm}^{2}$. This high deposition rate gave good properties in a $560 \mathrm{~nm}$-thick Nb47Ti film, where $T_{c}=8.95 \mathrm{~K}, \mu_{0} H_{c 2}=11.3$ $\mathrm{T}$ at $4.2 \mathrm{~K}$ (see Fig. 2), and the resistivity was $72 \mu \Omega-\mathrm{cm}$ at $T_{c}$. These values are close to the values of bulk Nb47Ti: 9.1 $\mathrm{K}, 11.1 \mathrm{~T}$, and $65 \mu \Omega-\mathrm{cm}$ respectively [3]. The layers are polycrystalline, as observed by TEM. No buffer layer was used; instead, the top and bottom layers are $\mathrm{Ti}$ pin layers.

A system of bridges was patterned by standard lithography techniques and wet etching. The bridge widths were measured by light microscopy using a calibrated length standard, and the effects of undercutting during the etching process were characterized by scanning electron microscopy. The multilayer thickness was determined by profilometer measurements. The bridge width used for the measurements was $35-60 \mu \mathrm{m}$, and the multilayer thickness was $1 \mu \mathrm{m}$. The voltage taps were $1 \mathrm{~cm}$ apart. Wires were then connected to the bridges by either using spring-loaded pins or by bonding them directly with indium. All critical current measurements using an apparatus described previously [6]. Measurements at $4.2 \mathrm{~K}$ were in liquid helium, and flowing $\mathrm{He}$ gas and a temperature controller were used at higher temperature. An electric field criterion of $0.1 \mu \mathrm{V} / \mathrm{cm}$ was used to determine the critical current. The value of $J_{c}$ was then determined by dividing the critical current by the cross-section of the bridge.

The superconducting properties of 2 of these multilayers are given focus in this paper, along with the Nb47Ti film described above. One had $d_{S}=45 \mathrm{~nm}$ and $d_{N}=15 \mathrm{~nm}$ $(45 / 15)$, and the other had $d_{S}=10 \mathrm{~nm}$ and $d_{N}=10 \mathrm{~nm}$ $(10 / 10)$. The $45 / 15$ film was studied because $d_{S}$ was much larger than $\xi$ but $d_{N}$ was thought to be less than $\xi_{N}$ [5], while the $10 / 10$ film was the smallest bilayer period which could be made with good adhesion and was thought to be fully proximity coupled. The critical temperature of these multilayers is shown in Table I. The value of $T_{c}$ was taken as the temperature at which resistance was zero for a constant current of $1 \mu \mathrm{A}$. The transitions were less than $0.1 \mathrm{~K}$ wide. The resistivity of the Ti layer $\rho_{N}$ and the proximity length are also given in Table I. These values were found by applying the parallel resistance law to the measured resistance, assuming that the resistivity of the $\mathrm{S}$ layers is equal to that of the Nb47Ti thin film. In previous work [5] the value of $\xi_{N}$ was determined by using the resistivity of a $0.23 \mathrm{~mm}$ diameter Ti wire, $\rho_{0}=5.6 \mu \Omega-\mathrm{cm}$. Since $\xi_{N}$ is proportional to

TABLE I

\begin{tabular}{llll}
\hline Multilayer & $T_{c}(\mathrm{~K})$ & $\rho_{N}(\mu \Omega-\mathrm{cm})$ & $\xi_{N}(\mathrm{~nm})$ \\
\hline $45 / 15$ & 8.45 & 56 & 10 \\
$10 / 10$ & 8.6 & 51 & 11 \\
\hline
\end{tabular}

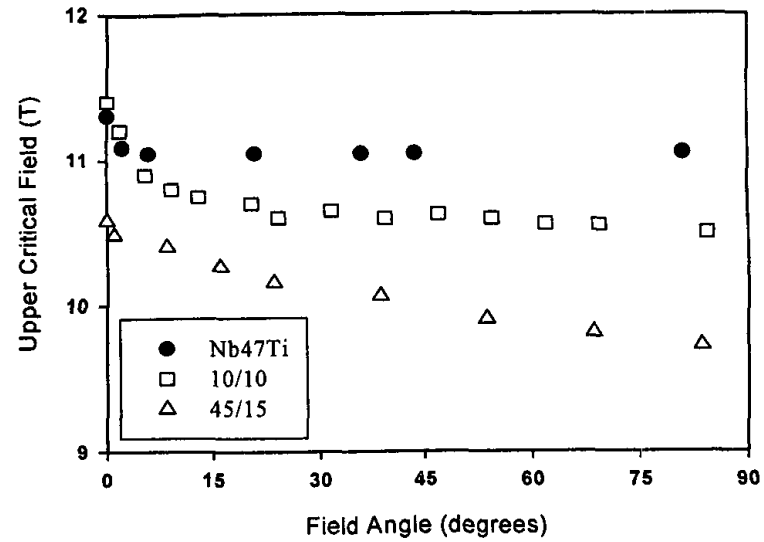

Fig. 1. Upper critical field as a function of the field angle for the $\mathrm{Nb47Ti}$ film and the multilayers. 0 degrees represents parallel field.

$\left(1 / \rho_{N}\right)^{1 / 2}$, the value in [5], $32 \mathrm{~nm}$, was multiplied by $\left(\rho_{0} / \rho_{N}\right)^{1 / 2}$ to obtain the values in Table $I$.

The upper critical field as a function of field angle $\theta$ at 4.2 $\mathrm{K}$ is shown in Fig. 1. The field at which the resistance was $1 \%$ of the normal-state resistance $R$ determined these data. A constant current of $1 \mu \mathrm{A}$ was applied while changing the field at fixed angle. The resistive transitions were very broad for the $\mathrm{Nb} 47 \mathrm{Ti}$ film and the multilayers, with approximately $2 \mathrm{~T}$ between $10 \%$ and $90 \%$ of $R$. Thus there is evidence of superconductivity even at $\sim 12 \mathrm{~T}, 4.2 \mathrm{~K}$, which may be due to the increased resistivity of the Nb47Ti layers. $H_{c 2}(\theta)$ has a small upturn and cusp near parallel field for both the $10 / 10$ multilayer and the $\mathrm{Nb} 47 \mathrm{Ti}$ film. Above $\sim 15^{\circ}$ the angular dependence is weak. Both of these samples exhibit high values of $\mu_{0} H_{c 2}$, reaching $11.3 \mathrm{~T}$ in parallel field and 10.8$11.0 \mathrm{~T}$ in perpendicular field. A moderate angular dependence is seen for the $45 / 15$ multilayer, with somewhat lower values for the upper critical field at all angles than for the thin film and the $10 / 10$ multilayer.

The critical current density as a function of parallel field at $4.2 \mathrm{~K}$ is shown in Fig. 2. The black symbols show $J_{c}$ data for $45 / 15$ and $10 / 10$. It can be seen that these data do not decrease monotonically with field as for the Nb47Ti film, shown by the dot-dash line, but have a series of peaks. This is more pronounced in the bulk pinning-force data, $F_{p}(H)=\mu_{0} H J_{c}(H)$, shown by the open symbols. The peaks are especially prominent in the $45 / 15$ data. Smoother plateaus are observed for the $10 / 10$ multilayer. The data for $10 / 10$ is limited to $-8 \mathrm{kA} / \mathrm{mm}^{2}$ because the sample quenched for the smallest bridge width tested, $35 \mu \mathrm{m}$. Apparently the value of $J_{c}$ increases between -2 and $\sim 4 \mathrm{~T}$ because stable current-voltage curves were acquired at $1.8,2$, and $2.25 \mathrm{~T}$. There is a sharp drop in the $J_{c}$ of the $10 / 10$ multilayer in the mid-field regime, from $\sim 8 \mathrm{kA} / \mathrm{mm}^{2}$ at $4 \mathrm{~T}$, to $\sim 5 \mathrm{kA} / \mathrm{mm}^{2}$ at 5 $\mathrm{T}$ and $\sim 2 \mathrm{kA} / \mathrm{mm}^{2}$ at $6 \mathrm{~T}$.

\section{DISCUSSION}

The body of literature about $\mathrm{Nb}-\mathrm{Ti}$ thin films and multilayers suggests that it is difficult to obtain superconducting properties comparable to those of bulk alloys by 


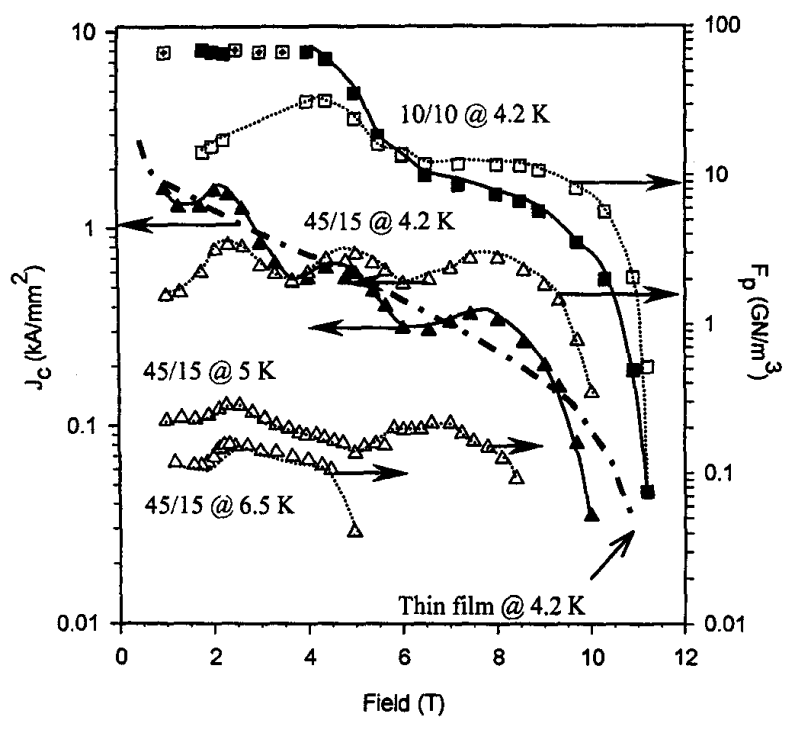

Fig. 2. Critical current density and bulk pinning force as a function of the applied parallel field. Critical current density for the $45 / 15$ and the $10 / 10$ multilayers are represented by the black triangles and black squares respectively. The white squares with crosses denote values at which a quench occurred. For comparison, the critical current density of the $560 \mathrm{~nm}$ $\mathrm{Nb47Ti}$ film is given by the dash-dotted line. Open symbols denote the bulk pinning force, as labeled near the curve.

sputtering. Generally the highest $T_{c}$ values are $8.6 \mathrm{~K}$ or less when the superconductor is Nb47Ti [6], [7], [14], and the resistivity is $75 \mu \Omega-\mathrm{cm}$ or higher [6], [14]. In a companion paper [15], it is shown that this may be due to the complicated nature of the sputtering process.

Nonetheless, the Nb47Ti film has better superconducting properties than reported previously. Using the same deposition parameters, good properties in multilayers are also obtained. The variation of the critical temperature between the thin film and the multilayers is less than the variation of $T_{c}$ for thin films made at the same power setting over the lifetime of the target [15]. This suggests that there is no clear evidence for suppression of $T_{c}$ by the proximity effect. By contrast, $T_{c}$ was strongly suppressed for $\mathrm{Nb} 47 \mathrm{Ti}$ multilayers with pure $\mathrm{Cu}$ [6] or $\mathrm{Cu}$-alloy [10] pins. In that case 45/15 multilayers had a $T_{c}$ between 8 and $8.2 \mathrm{~K}$, and $10 / 10$ multilayers had $T_{c}<6.5 \mathrm{~K}$. The apparent lack of proximity effect suppression is also indicated by the upper critical field data. For the most part, $H_{c 2}(\theta)$ is not strong. The small cusp in parallel field for the $10 / 10$ multilayer indicates a somewhat enhanced $H_{c 2}$ value of $11.4 \mathrm{~T}$, which could be due to a dimensional crossover of the $S$ layers [15] or surface superconductivity. These data are in contrast to data for $\mathrm{Cu}-$ alloy [6], [10] and $\mathrm{Nb}$ [11] pin layers, which exhibit a change by a 1-4 $\mathrm{T}$ in the value of $H_{c 2}$ between parallel and perpendicular field.

The high resistivity of the Ti layers, $\sim 50 \mu \Omega-\mathrm{cm}$, may be beneficial. The proximity length of pure $\mathrm{Nb}$, pure $\mathrm{Ti}$, and $\mathrm{Nb}$-alloys were compared in [5]. This showed that pure $\mathrm{Ti}$ had a similar value of $\xi_{N}$ as $\mathrm{Nb} 10 \mathrm{wt} . \% \mathrm{~W}$. However, in the present case the resistivity is much higher than the value used in [5], resulting in an even smaller value of $\xi_{N}$, about $10 \mathrm{~nm}$. More recent work [10] argues that the proximity length of $\mathrm{Cu} 30 \mathrm{at} \% \mathrm{Ni}$, a strong alloy of copper, is greater than $20 \mathrm{~nm}$. Thus, pure $\mathrm{Ti}$ is very effective at limiting the extent of the superconducting pair function, and the possible deleterious properties of alloying elements are not present.

The behavior of $J_{c}$ and $F_{p}$ are interesting. The pinning force for $45 / 15$ shows several peaks at $4.2 \mathrm{~K}, 5 \mathrm{~K}$, and $6.5 \mathrm{~K}$. At $4.2 \mathrm{~K}$ the peaks correspond to $a_{0} \approx 30,20$, and $15 \mathrm{~nm}$ at $2.2,5$, and $7.8 \mathrm{~T}$ respectively. The $2.2 \mathrm{~T}$ peak is also present at $5 \mathrm{~K}$ and $6.5 \mathrm{~K}$, but the high-field peaks become broad and move to $\sim 6 \mathrm{~T}$ in the $5 \mathrm{~K}$ data. The fact that the $2.2 \mathrm{~T}$ peak is not dependent on temperature suggests that it is due to a matching effect, $\Lambda=2 a_{0}$. The other peaks may obey similar configurations for $d_{S}, \Lambda$, and $a_{0}$. In this scenario, the first peak corresponds to having 1 flux line row in the center of the $S$ layers and an adjacent row in the $N$ layers; the second to 2 rows in the $\mathrm{S}$ layer and 1 in the $\mathrm{N}$ layer; and so on. Similar phenomena have been studied in other layered superconductors [16].

The $10 / 10$ multilayer demonstrates much stronger pinning below $\sim 5 \mathrm{~T}$. In this case $J_{c}$ approaches $8 \mathrm{kA} / \mathrm{mm}^{2}$ at $4 \mathrm{~T}$ and below, and 2 plateaus appear for $J_{c}(H)$. A steep drop separates the plateaus, occurring when $a_{0}=\Lambda(\sim 5 \mathrm{~T})$. The plateaus might again be explained by a matching effect; unfortunately, this sample was destroyed after one of the lowfield measurements, and data at higher temperature could not be obtained to support this hypothesis. The data suggest that smaller bilayer periods will not only give higher critical current density at low field, but also shift the plateaus to higher flux densities. The critical current density at $5 \mathrm{~T}, \sim 5$ $\mathrm{kA} / \mathrm{mm}^{2}$, and at $8 \mathrm{~T}, \sim 1.5 \mathrm{kA} / \mathrm{mm}^{2}$, are about the same as the data for a Nb47Ti tape with $\alpha$-Ti precipitates which were aligned to within $\sim 10^{\circ}$ of the tape face. The similarity of these data may reflect a balance between 3 effects: Although the pin number density is somewhat higher in the tape (1 precipitate per $5-10 \mathrm{~nm})$ than in the film $(1$ pin per $20 \mathrm{~nm})$, the distribution of the precipitate thickness of the tape is broader than the distribution of the pin layer thickness in the multilayer, and the volume fraction of pins in the tape $(\sim 20 \%)$ is less than that of the multilayer (50\%).

The similarity between the tape and the multilayer also suggests that the flux lines are not strongly affected by variations in the layer orientation. To test this hypothesis, we analyzed a $10 \mathrm{~nm} \mathrm{Nb47Ti} / 3 \mathrm{~nm}$ Ti multilayer using TEM. This multilayer was made during the same run as the $10 / 10$ multilayer, however it exhibited visible signs of stress during subsequent processing. As shown in Fig. 4, columnar grains grow through several layers and cause a local misorientation of the layers of $\sim 5^{\circ}$. Similar TEM observations were made in [8]. This perturbation is somewhat larger than the angular variation of $J_{c}$, which had a cusp at $\theta=0^{\circ}$ and a width at halfmaximum of $\sim 3^{\circ}$. These data suggest that the flux lines can follow the contours of the pin layers without a significant reduction of the pinning force.

A new limitation of $J_{c}$ of $\mathrm{Nb}-\mathrm{Ti} / \mathrm{Ti}$ multilayers is poor stability. In contrast to $\mathrm{Cu}$ and $\mathrm{Nb}$ pin layers, which are good conductors of heat, the thermal conductivity of $\mathrm{Ti}$ is about the same as $\mathrm{Nb} 47 \mathrm{Ti}$. This suggests that heat cannot escape 


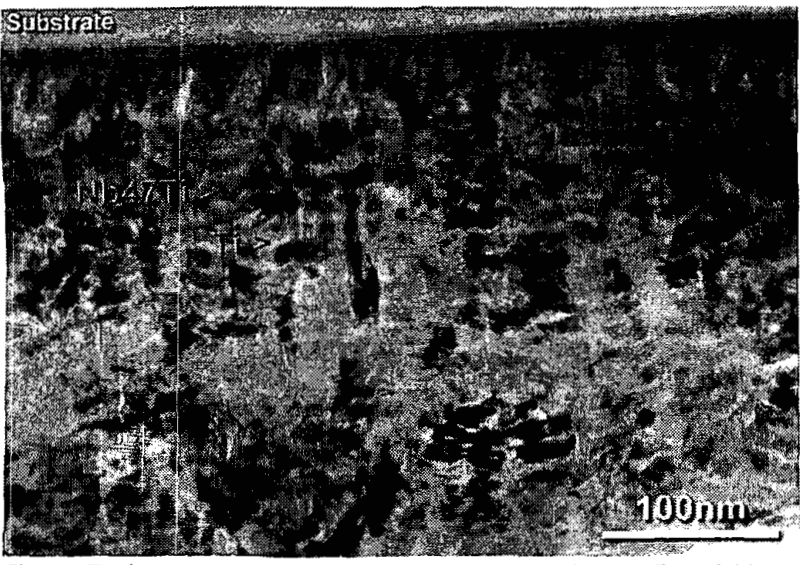

Fig. 4. Tranismission electron microscopy image of a $10 \mathrm{~nm} \mathrm{Nb47Ti} / 3 \mathrm{~nm}$ Ti multilayer. The sapphire substrate is at the top edge of the photograph. The bright regions are $\mathrm{Ti}$, while the darker gray regions are $\mathrm{Nb47Ti}$. The nearly black regions are grains which strongly diffract the electron beam. The layer structure of the multilayer is evident as horizontal features, while grain boundaries run vertically. Precipitates may also be present, as indicated by the vertical white streaks in the lower left corner.

between the superconducting layers. It was also noted in Fig. 3 that the $J_{c}$ data was limited to $\sim 8 \mathrm{kA} / \mathrm{mm}^{2}$ by a quench at low fields. This may be due to a lack of adiabatic stability. By using a standard textbook expression and established values of the specific heat and density of $\mathrm{Nb} 47 \mathrm{Ti}$ [17], the critical bridge width for $8 \mathrm{kA} / \mathrm{mm}^{2}$ current density can be estimated as $-30 \mu \mathrm{m}$. In addition, dynamic stability is also poor, where the Stekly number is above 100 [18]. A solution to these stability problems may require the construction of "multifilamentary" bridges, in which 1-10 $\mu \mathrm{m}$ lines are patterned into the multilayer and are subsequently coated with a thick layer of copper.

The critical current density shown in Fig. 3 is not as high as that reported in [7], despite having similar layer dimensions (19/5 in [7]; $10 / 10$ in this paper) and good superconducting properties. However, there are several important differences between the 2 experiments which make the results difficult to compare. In [7], the multilayers contained 10 bilayers and were coated by $50 \mathrm{~nm}$-thick $\mathrm{Nb}$ layers on the top and bottom, so it is difficult to assess the critical temperature of the multilayer and how much current might be carried by the $\mathrm{Nb}$. The electric field criterion used to determine the critical current in [7] was $50 \mu \mathrm{V} / \mathrm{cm}$; here it is $0.1 \mu \mathrm{V} / \mathrm{cm}$. Given that the current-voltage data obeys a power law with an exponent (n-value) of $\sim 30$, the different criteria can give a difference in $J_{c}$ of $\sim 15 \%$. The bridge width in [7] was $3 \mu \mathrm{m}$, which along with the $\mathrm{Nb}$ coating would enhance the stability of the multilayer substantially. These differences underscore the variety of different ways that such experiments can be done, and more systematic study of multilayers and their associated physics is needed.

\section{CONCLUSIONS}

In conclusion, multilayers of $\mathrm{Nb} 47 \mathrm{Ti}$ and $\mathrm{Ti}$ were discussed and compared to a $560 \mathrm{~nm}$-thick Nb47Ti film. The film exhibited superconducting properties which were close to those for bulk specimens. Similar parameters were obtained in the multilayers by using the same deposition parameters. The multilayer with $10 \mathrm{~nm} \mathrm{Nb47Ti}$ and $10 \mathrm{~nm} \mathrm{Ti}$ had virtually no suppression of $T_{c}$ and $H_{c 2}$ despite the expectation of strong proximity coupling. This occurred because the proximity length of Ti was $\sim 10 \mathrm{~nm}$. The critical current density of a $45 \mathrm{~nm} / 15 \mathrm{~nm}$ multilayer exhibited matching effects. The $10 / 10$ multilayer achieved $J_{c}$ values comparable to those of a Nb47Ti tape conductor, reaching 5 $\mathrm{kA} / \mathrm{mm}^{2}$ at $5 \mathrm{~T}, 4.2 \mathrm{~K}$. The $10 / 10$ multilayer also approached the limit of adiabatic stability, which resulted in some $J_{c}$ data being limited by a quench. Methods to improve the stability were discussed. Also, the critical current density data was compared to a previous result [7], and several differences in the experiments were found.

\section{ACKNOWLEDGMENT}

Discussions with M. Tachiki and A. Gurevich are gratefully acknowledged.

\section{REFERENCES}

[1] P.J. Lee and D.C. Larbalestier, Acta Metall., vol. 35, pp. 25232536,1987

[2] L.D. Cooley, P.J. Lee, and D.C. Larbalestier, Phys. Rev. B,vol. 53 , pp. 6638-6652, 1996.

[3] C. Meingast, P.J. Lee, and D.C. Larbalestier, J. Aspl. Phys., vol. 66, pp. 5962-5970, 1989.

[4] K. Matsumoto, Y. Tanaka, K. Yamafuji, M. Iwakuma, and T. Matsushita, IEEE Trans. Appl. Supercond., vol. 5, pp. 1362-1365, 1993.

[5] R.W. Heussner, C.B. Nunes, L.D. Cooley, and D.C. Larbalestier, IEEE Trans. Appl. Supercond., vol. 7, pp. 1142-1 145, 1997.

[6] E. Kadyrov, A. Gurevich, and D.C. Larbalestier, A.ppl. Phys. Lett., vol. 68, pp. 1567-1569, 1996.

[7] J.D. McCambridge et al., IEEE Trans Appl. Supercond., vol. 7, pp. 1134-1137, 1997.

[8] W.H. Warnes, K.J. Faase, and N.A. Norris, A.dv. Cryo. Eng. (Materials), vol. 42B, pp. 1143-1150, 1996.

[9] The bridge width reported in ref. [6], $50 \mu \mathrm{m}$, was in error. The correct value is $150 \mu \mathrm{m}$.

[10] L.D. Cooley, C.D. Hawes, and D.C. Larbalestier, to be published.

[11] Y. Kuwasawa, T. Tosaka, A. Uchiyama, S. Matude, and S. Nakano, Physica C, vol. 175, pp. 187-191, 1991.

[12] L.D. Cooley, P.D. Jablonski, P.J. Lee, and D.C. Larbalestier, Appl. Phys. Lett., vol. 58, pp. 2984-2986, 1991.

[13] C.D. Hawes, L.D. Cooley, and D.C. Larbalestier, paper MFA-01, this conference.

[14] G. Stejic, A. Gurevich, E. Kadyrov, D. Christen, R. Joynt, and D.C. Larbalestier, Phys. Rev. B, vol. 49, pp. 1274-1288, 1994.

[15] S. Takahashi and M. Tachiki, Phys. Rev. B, vol, 33, pp. 4620-4631, 1986.

[16] S.H. Brongersma, E. Verweij, N.J. Koeman, D.G. deGroot, R. Griessen, and B.I. Ivlev, Phys. Rev. Lett., vol. 71, pp. 2319-2322, 1993.

[17] M.N. Wilson, Superconducting Magnets, Oxford: Clarendon, 1986.

[18] A.V. Gurevich and R.G. Mints, Rev. Mod. Phys.,vol. 59, pp. 941999, 1987. 